\title{
LA UNIVERSIDAD CUBANA Y SUS RETOS ANTE LOS NUEVOS ACTORES DE LA ECONOMÍA NACIONAL
}

\author{
THE CUBAN UNIVERSITY AND HER CHALLENGE CONSIDERING \\ THE NEW NATIONAL ECONOMIC SUBJECTS
}

\author{
LUIS PÉREZ OROZCO \\ Universidad de Matanzas, Cuba
}

\begin{abstract}
Resumen: En Cuba se lleva a cabo actualmente un proceso de actualización económica que incluye el redimensionamiento al trabajo por cuenta propia y a las cooperativas no agropecuarias como sujetos de la actividad mercantil. Sin embargo, tales actores se desempeñan en circunstancias en las que todavía predominan los tabúes respecto a ellos. En ese contexto, la Universidad cubana se encuentra ante un nuevo panorama y en ocasiones no encuentra las formas de interactuar con esos actores económicos. La transferencia de tecnología y conocimientos mutuos son a la postre las áreas fundamentales en la que la relación Universidad-sector productivo puede ser favorable para ambos. El intercambio puede ser posible mediante el marco conceptual que brinda el modelo de la Triple Hélice. La presente investigación argumenta la factibilidad de la interrelación de la institución universitaria cubana y los más recientes actores de la economía nacional mediante el ya mencionado modelo.
\end{abstract}

Palabras clave: Universidad; trabajo por cuenta propia; cooperativas no agropecuarias; Cuba.

\begin{abstract}
In Cuba, a process of economic updating is currently carried out that includes resizing to self-employment and non-agricultural cooperatives as subjects of commercial activity. However, such actors perform in circumstances in which the taboos against them still predominate. In this context, the Cuban University is facing a new panorama and sometimes does not find ways to interact with these economic actors. The transfer of technology and mutual knowledge are ultimately the fundamental areas in which the University-productive sector relationship can be favorable for both. The exchange can be possible through the conceptual framework provided by the Triple Helix model. The present investigation argues the feasibility of the interrelation of the Cuban university institution and the most recent actors of the national economy through the aforementioned model.
\end{abstract}

Keywords: University; self-employment; non-agricultural cooperatives; Cuba.

SUMARIO: 1. UNA BREVE APROXIMACIÓN A LA SITUACIÓN ACTUAL DE LOS NUEVOS SUJETOS DE LA ECONOMÍA CUBANA. 2. LA DINÁMICA ECONÓMICA DE CUBA Y PERSPECTIVAS DE LAS UNIVERSIDADES. 3. HACIA UNA NUEVA RELACIÓN UNIVERSIDAD-SECTOR PRODUCTIVO. 4. EL MODELO DE LA TRIPLE HÉLICE Y LOS VÍNCULOS UNIVERSIDAD-GOBIERNO-SECTOR PRODUCTIVO. 5. A MODO DE CONCLUSIONES.

\section{INTRODUCCIÓN}

La producción y aplicación de conocimiento es el motor que impulsa la economía, otorgando una ventaja competitiva a los países que lo jerarquizan. La im- 
portancia de este recurso está dada en que se constituye en una posibilidad legítima que tiene toda sociedad que desee integrarse con éxito económicamente. Los países subdesarrollados se enfrentan a múltiples desafíos. Estos abarcan áreas como proporcionar empleos y alimentos a la sociedad, entre otros. Entre las formas de enfrentar estos desafíos está emplear una de las instituciones más eficientes: las organizaciones, en el caso que ocupa esta investigación, la Universidad. Para que en países como Cuba pueda darse esta integración resulta necesario que los Centros de Educación Superior sean el lugar donde, por historia y condiciones, se debe generar y difundir el conocimiento.

A medida que el acceso a la Universidad se hizo masivo, surgieron problemas en cuanto a la manera de financiar y concebir sus actividades y si estas sólo se deben regir por los aportes del Estado. En un mundo regido por la internacionalización de las relaciones comerciales, se considera que el vínculo entre Universidad y empresa también debe ser el centro del debate y eje del desarrollo económico y social. La intención de esta investigación es argumentar que la relación entre la Universidad y los nuevos agentes económicos en Cuba es válida para el mejoramiento de esa institución.

\section{UNA BREVE APROXIMACIÓN A LA SITUACIÓN ACTUAL DE LOS NUEVOS SUJETOS DE LA ECONOMÍA CUBANA}

Desde el 2011 se ha puesto en marcha en Cuba un proceso de cambios importantes que afectan a ámbitos centrales del modelo económico previamente existente. Las transformaciones de naturaleza estructural, incluyendo también reformas en los mecanismos de gestión, y en términos de organización económica y administrativa, implican retos trascendentes desde el punto de vista institucional.

La constatación de dichas deficiencias estructurales llevó a las máximas autoridades del país a plantear la necesidad de un importante proceso de transformaciones, en las formas de gestión y en la política económica nacional, bajo la denominación de "actualización del modelo económico," el cual fue aprobado en el VI Congreso del Partido Comunista de Cuba (PCC) en 2011. Como parte de dicho programa, desde ese momento se han introducido múltiples modificaciones en la economía y en el sistema legal cubano.

Dentro de las modificaciones se destacan aquellas que tienen como propósito el redimensionamiento de la economía nacional en términos de empleo, estructura de la propiedad, y escala de producción. Se abren más espacios y se promueve un escenario en el cual las consideraciones de mercado se van incorporando en el diseño de políticas públicas para la regulación de la economía. Al mismo tiempo, y como resultado de las anteriores dinámicas, empiezan a consolidarse formas no estatales de propiedad y gestión, incluyendo la proliferación de nuevos emprendimientos que pueden ya clasificarse como micro, pequeñas y medianas empresas.

Dentro de las formas de organización productiva que se reestructuran en Cuba, además de las modalidades de inversión extranjera, aparecen el trabajo por cuenta propia y el cooperativismo. 
A partir del Decreto-Ley No. 305 y No. 306, ambos de 2012, se abrió el camino para la gestión de cooperativas no agropecuarias en Cuba. La nueva etapa del desarrollo económico, como recogieron los Lineamientos de la Política Económica y Social, ha colocado al cooperativismo entre las principales formas de producción a poner en práctica.

Según el artículo 2.1 del Decreto-Ley No. 305: "La cooperativa es una organización con fines económicos y sociales, que se constituye voluntariamente sobre la base del aporte de bienes y derechos y se sustenta en el trabajo de sus socios, cuyo objetivo general es la producción de bienes y la prestación de servicios mediante la gestión colectiva, para la satisfacción del interés social y el de los socios".

Las cooperativas no agropecuarias existentes en el país (19 de origen estatal) agrupan a más de 460 socios. E1 $99 \%$ de los presidentes esas organizaciones se mantienen en sus cargos. Han alcanzado ingresos superiores a los 204000000 pesos, con una utilidad de 59.1 millones (todas generaron ganancias). El promedio del anticipo a esos trabajadores es de 3200 pesos por socios. Además, han cumplido sus obligaciones fiscales sin retrasos, contribuyen como complemento tecnológico en muchos procesos productivos y en las dinámicas de las empresas estatales; se integraron a programas como el ahorro de agua y la construcción de viviendas; rescatan servicios, apoyan programas de desarrollo comunitario y aportan al mantenimiento de reparación de inmuebles sociales, además de sustituir importaciones.

A principios de 2013 el Vicepresidente Marino Murillo Jorge propuso al Consejo de Ministros la aprobación del primer grupo de 124 cooperativas no agropecuarias que se iniciaron en diversas actividades: 99 correspondían a mercados agropecuarios, cinco para el servicio de transporte de pasajeros, seis de servicios auxiliares de transporte, dos de reciclaje de desechos, y 12 de actividades vinculadas a la construcción. De este primer grupo de cooperativas, 112 surgen del sector estatal y 12 del no estatal, estas últimas constituidas fundamentalmente por trabajadores por cuenta propia.

En opinión de las autoridades gubernamentales, son satisfactorios los resultados que registran estas nuevas formas de gestión no estatal. Al respecto, constatan que los cooperativistas se sienten contentos, y como fruto de su gestión son bien remunerados; ha mejorado la calidad de los servicios; hay una presencia más estable (con mayor variedad y selección) de los productos en los mercados. Igualmente se observa una sostenible mejora en la imagen de los establecimientos arrendados. Según la Oficina Nacional de Estadísticas e Información (ONEI), al cierre del 2016 funcionaban en la Isla 367 cooperativas de nuevo tipo, superior en poco más de una veintena a las existentes en 2014 (345). Tal ritmo de crecimiento es el menor registrado desde que el país impulsó en 2013 ese segmento del sector no estatal, con la autorización de 198 organizaciones colectivas y otras 147 al año siguiente. Los sectores del comercio, gastronomía, servicios técnicos y personales; construcción; e industria, concentran la mayor cantidad. La mayoría ubicada en las provincias La Habana, Matanzas, Pinar del Río y Artemisa, aunque poseen representación en el resto del país.

En el Consejo de Ministros los informes presentados esgrimen criterios positivos respecto a la efíciencia económica del experimento; al tiempo que se destacan 
aspectos negativos que atañen a la aplicación y comprensión de su carácter experimental. Frente a esa dualidad se resolvió no masificar la creación de cooperativas, priorizar la consolidación de las que existen y postergar la propuesta de cooperativas de segundo grado.

Además, hasta el año 2015 la banca cubana había otorgado, en créditos, 223000 000 de pesos en moneda nacional para respaldar el progreso de las cooperativas no agropecuarias. Alrededor de 324 organizaciones colectivas se han beneficiado con esos préstamos, con predominio de las solicitudes para capital de trabajo y la ejecución de inversiones.

Aunque con el surgimiento de las cooperativas no agropecuarias afloran beneficios asociados, por ejemplo, al desarrollo de las comunidades, a la sustitución de importaciones, al mantenimiento y la reparación de inmuebles sociales, a los encadenamientos productivos con empresas estatales, así como al rescate de servicios perdidos; existen problemas e insatisfacciones que limitan esta forma de gestión no estatal. Debilidades que, por una parte, están relacionadas con la bisoñez del proceso.

También, actualmente circulan en el país dos monedas (CUP y CUC) y dos tipos de cambio distintos (uno para las personas jurídicas, y otro para las naturales). Es una consecuencia directa de lo anterior que en Cuba los precios minoristas no son continuidad de los mayoristas.

También, desde octubre de 2010, se inicia un relanzamiento del sector cuentapropista que consiste, en el descongelamiento de un grupo de categorías de las cuales no se efectuaban autorizaciones. Actualmente la lista de licencias autorizadas comprende 123 actividades Se establece también un marco regulatorio más flexible que el precedente ${ }^{1}$.

Los nuevos productores y comercializadores privados pueden comercializar sus bienes y servicios con entidades estatales, así como contratar fuerza de trabajo fuera del marco familiar. Contribuyen y se benefician de la seguridad social. Tienen acceso al crédito bancario. Pueden arrendar locales o activos del Estado u otros ciudadanos. Una persona tiene la facilidad de disponer de varias licencias para ejercer más de una actividad por cuenta propia, tanto en su municipio de procedencia como en cualquier lugar del país. Se elimina la restricción para arrendar el total de una casa o departamento, emplear el arrendamiento de habitaciones por horas y utilizar inmuebles asignados o reparados por el Estado en el último decenio. Se prescinde del requisito de ser jubilado o tener algún vínculo laboral para acceder a esta forma de empleo.

En este contexto, las modificaciones institucionales, al tiempo que resultado de los cambios enunciados, tienen que dar respuesta a las necesidades del entramado empresarial del país, y coadyuvar al progreso y bienestar social. Dicho en otras palabras, el proceso de transformación económica en curso exige mayores cambios estructurales que amplíen y regulen el espacio de acción del mercado. Pueden exis-

La Gaceta Oficial Extraordinaria No. 35 de 10 de julio de 2018 abarca un conjunto de regulaciones sobre el trabajo por cuenta propia en Cuba, que deroga las anteriores normativas al respecto. 
tir varias propuestas al respecto, las autoridades cubanas pudieran considerar como prioridad en las futuras modificaciones algunas de las que a continuación se detallan.

El fortalecimiento de la institucionalidad para promover un régimen de seguridad jurídica para todos los agentes económicos, para lo cual resulta necesario el diseño, discusión y aprobación de una Ley de Empresas. Dicho instrumento legal y sus normas complementarias deben tomar nota de la gran dispersión y heterogeneidad del entramado de empresas existentes y las que están emergiendo como parte del proceso de cambios, la mayoría de ellas con muy reducido nivel de desarrollo tecnológico, limitaciones en el acceso a las tecnologías de la información y las comunicaciones (TICs), pocos avances en términos de asociatividad, y en general baja productividad. Al mismo tiempo, debería avanzarse en la aprobación de las disposiciones necesarias para consolidar entidades que presten servicios de apoyo al sector empresarial (legales, de consultoría, auditoría, contabilidad, investigación de mercados, diseño de productos y procesos, por solo mencionar algunas).

En las condiciones actuales parece sensato asumir que las perspectivas de desarrollo del país estarán directamente vinculadas a una mayor y mejor integración en los mercados internacionales, por lo que urge construir políticas que favorezcan este proceso y permitan manejar adecuadamente los costos asociados ${ }^{2}$. Ello requiere una reforma de la institucionalidad que regula el comercio exterior cubano, con vistas a flexibilizar el nivel de concentración y centralización que todavía tienen las operaciones comerciales externas del país, y permitir el acceso de entidades no estatales a los mercados de exportación e importación.

En un país como Cuba, el establecimiento de una política de competencia, así como sus correspondientes normas e instancias de aplicación resulta imprescindible. La aparición de nuevas formas de propiedad y de nuevos agentes económicos; la modificación en los mecanismos de regulación y control estatal sobre la economía; los cambios en la planificación y administración; las nuevas atribuciones que empiezan a otorgarse a las empresas estatales; la presencia de diversos agentes en las operaciones del comercio exterior, y la descentralización en los procesos para la determinación de los precios (mayoristas y minoristas), constituyen transformaciones económicas que han propiciado la aparición de mercados y de espacios de competencia en el entorno cubano ${ }^{3}$.

Otro ámbito de la política pública a desarrollar por el Estado en estas nuevas condiciones es el relativo a la política regional, y consecuentemente la aprobación del conjunto de normas que garanticen los derechos de los territorios, en el marco de un proceso de descentralización administrativa. Dichas normas deberían coadyuvar

R. TORRES PÉREZ, “Algunas contradicciones del desarrollo económico cubano contemporáneo”, en O. E. PÉREZ y R. TORRES (Comps.), Miradas a la Economía cubana. Entre la eficiencia económica y la equidad social, Editorial Caminos, La Habana, 2013, p. 38.

3 La competencia en Cuba expresada en la creciente diversidad de entidades que realizan la misma actividad, ha determinado la aparición de una evidente diferenciación económica, que se expresa en términos de precios, de calidad, de garantías y de servicios posventa, todos los cuales constituyen indicadores típicos de relaciones de concurrencia. 
al logro de una distribución más homogénea de las capacidades productivas a lo largo del territorio nacional.

En definitiva, el avance de los ajustes, la transformación económica y sus inevitables modificaciones, abre nuevas preocupaciones teóricas y prácticas acerca de las desigualdades territoriales en el país que por ahora no parecen detenerse ${ }^{4}$. Las razones por las que el traspaso de poderes presupuestarios a los gobiernos subcentrales podría impulsar la actividad económica son muy variadas ${ }^{5}$. Obviamente, para que se desplieguen los beneficios propios de la descentralización fiscal es importante que exista un adecuado diseño de las instituciones a través de las cuales se canalizan las relaciones intergubernamentales.

La actividad innovadora que incluye a la investigación básica como parte fundamental de la actividad investigadora y tiene los rasgos propios de un "bien público;" la protección de los recursos, bienes y servicios; así como el cuidado y la promoción del patrimonio cultural son áreas estratégicas que no pueden ser marginadas dentro de las prioridades estatales y para las cuales se requiere el diseño y desarrollo de complejas, pero eficientes instituciones.

\section{LA DINÁMICA ECONÓMICA DE CUBA Y PERSPECTIVAS DE LAS UNIVERSIDADES}

Dentro de los esfuerzos del país por generar desarrollo económico, se ha hecho especial énfasis en incentivar la investigación y la innovación, proceso que ha sido guiado por la teoría y la investigación empírica que proveen los modelos de crecimiento endógeno. Estos destacan el papel central de la acumulación y difusión de conocimiento al crecimiento de la producción nacional.

La evolución de los problemas y del contexto global, el perfeccionamiento de la Universidad tradicional de docencia e investigación hasta la actual, la ampliación de la frontera tecnológica y la profundización de la ciencia básica en todos los procesos y ámbitos de la sociedad ha generado que las esferas que produzcan mayor contribución al desarrollo modifiquen su radio de acción. De esta forma las necesidades que genera la "sociedad del conocimiento" llevan a que cada uno de los actores de las relaciones trilaterales de la Universidad, el Estado y la empresa desempeñen un rol que desborda su misión tradicional ${ }^{6}$.

\footnotetext{
L. ÍNIIGUEZ ROJAS, “Desigualdades territoriales y ajustes económicos en Cuba”, en O. E. PÉREZ y R. TORRES (Comps.), Miradas a la Economía..., cit., p. 115.

5 De todas formas, la descentralización fiscal no siempre implica una provisión más eficiente de los servicios. Hay algunos casos en los que el suministro uniforme a nivel nacional tiene ventajas que deben ser consideradas.

6 Las Universidades frecuentemente al asumir funciones empresariales se comportan como emprendedores, interpretando su investigación y docencia en nuevas formas y ayudando a la creación de novedosas empresas en centros de incubación. El Tecnológico de Monterrey desarrolla un modelo de incubación de empresas basado en cuatro etapas: Selección, Pre-incubación, Incubación y Post-incubación. Estrategia que comienza con la selección del proyecto, pasa por la creación del modelo y el plan de negocio, la definición de Indicadores Claves de Desempeño, así como
} 
En Cuba los Lineamientos de la Política Económica y Social del Partido y la Revolución para el periodo 2016-2021 aprobados en el VII Congreso del PCC, respaldan este proceso. El Lineamiento No. 13 plantea "priorizar y continuar avanzando en el logro del ciclo completo de producción mediante los encadenamientos productivos entre organizaciones que desarrollan actividades productivas, de servicios y de ciencia, tecnología e innovación, incluidas las universidades, que garanticen el desarrollo rápido y eficaz de nuevos productos y servicios, con estándares de calidad apropiados, que incorporen los resultados de la investigación científica e innovación tecnológica, e integren la gestión de comercialización interna y externa".

El Lineamiento No. 125 dispone "consolidar el cumplimiento de la responsabilidad de los organismos, entidades, consejos de la administración y otros actores económicos, en la formación y desarrollo de la fuerza de trabajo calificada. Actualizar los programas de formación e investigación de las universidades en función de las necesidades del desarrollo, de las nuevas tecnologías y de la actualización del Modelo Económico y Social".

Por su parte el Plan Nacional de desarrollo económico y social hasta 2030: propuesta de visión de la nación, ejes y sectores económicos estratégicos establece como eje estratégico el potencial humano, la ciencia, la tecnología e innovación 7 .

el desarrollo del plan de negocios en tiempo real hasta un período de seguimiento y adaptación de los objetivos y aprovechamiento de áreas de oportunidad. En total la estrategia de seguimiento y asesoría puede durar hasta tres años, período en el cual la Universidad es la que permite la creación y perdurabilidad de las empresas.

El Plan Nacional de desarrollo económico y social hasta 2030 en cuanto al eje estratégico sobre el potencial humano, ciencia, tecnología e innovación propone priorizar los sectores y actividades en que la ciencia, la tecnología y la innovación tengan mayor y más inmediato impacto en el crecimiento económico. Promover el cierre del ciclo científico-productivo, mediante el aseguramiento de una estrecha interacción entre la generación del nuevo conocimiento y la producción de bienes y servicios en función de la integración de los resultados a las cadenas productivas. Adecuar el marco jurídico y regulatorio de la ciencia, la tecnología y la innovación al proceso de actualización del modelo económico, de manera que se logre agilidad, flexibilidad, eficiencia en los mecanismos organizativos y económico-financieros para la materialización del impacto de estas actividades en el desarrollo económico y social. Potenciar la innovación y su generalización en el sector productivo, utilizando los incentivos necesarios, para que constituya un componente esencial del incremento de la eficiencia económica, la competitividad de las producciones y el cambio tecnológico. Desarrollar procesos y tecnologías autóctonas que garanticen una utilización adecuada de las materias primas, materiales y recursos naturales del país y contribuyan a la soberanía tecnológica. Incrementar de forma sostenida el monto del financiamiento de la actividad de ciencia, tecnología e innovación y su crecimiento proporcional en relación con el producto interno bruto (PIB). Fortalecer las capacidades de prospección y vigilancia tecnológica, así como la protección de la propiedad intelectual (derechos de autor y propiedad industrial) en Cuba y en los principales mercados externos. Garantizar el desarrollo de las Universidades, sus recursos humanos e infraestructura para impulsar la formación del potencial humano de alta calificación y la generación de nuevos conocimientos. Diseñar programas de estudio y potenciar el otorgamiento de becas de formación de estudios avanzados para jóvenes talentos en las Universidades e institutos tecnológicos que respondan a requerimientos de introducción de tecnologías de avanzada, en correspondencia con las demandas del desarrollo económico y social. Establecer estímulos para la protección y estabilidad del personal de más alta calificación, mayor experiencia, jóvenes talentos y de difícil 
Además de tener en cuenta esas orientaciones del Estado y el Gobierno, se debe considerar que el sistema de Educación Superior en Cuba se desenvuelve en algunas particularidades: el Estado dedica hasta tres años de preparación a los egresados mediante el Servicio Social, los nuevos actores de la economía aumentan cada vez más su presencia, la distribución del gasto universitario se caracteriza por montos dedicados a personal y montos a equipamiento y mejora de la calidad de los procesos vitales: docencia, investigación y extensión ${ }^{8}$, la fuga de los recursos humanos de mayor calificación hacia países desarrollados y dentro del territorio nacional, hacia otros sectores distintos a los estudiados, los aportes de la Educación Superior para la renovación y mejora de la capacidad industrial y servicios, así como el aumento del respeto hacia el valor de la Educación Superior que experimenta la sociedad cubana debido a los resultados obtenidos en concursos de habilidades y aporte al desarrollo científico.

Por los hechos anteriormente expresados, entre otros, es que se debe analizar el objeto relación Universidad-sociedad como sistema, es decir, sin absolutizar el replanteo del subsistema Educación Superior desde fuera, ni desde adentro.

Según la Resolución No. 2 de 21 de junio de 2018 Reglamento para el Trabajo Docente y Metodológico en la Educación Superior ${ }^{9}$, la formación de los profesionales de ese nivel de enseñanza es el proceso que, de modo consciente y sobre bases científicas, se desarrolla en las instituciones de educación superior para garantizar la preparación integral de los estudiantes universitarios, que se concreta en una sólida formación científico-técnica, humanística y de altos valores ideológicos, políticos, éticos y estéticos, con el fin de lograr profesionales revolucionarios, cultos, competentes, independientes y creadores, para que puedan desempeñarse exitosamente en los diversos sectores de la economía y de la sociedad en general ${ }^{10}$.

El proceso curricular incluye la actividad docente educativa, y la extracurricularidad está dada por acciones de impacto social o extensionismo universitario por solo citar algunas esferas, constituyendo elementos de vital importancia para la formación integral de los estudiantes ${ }^{11}$.

Las carreras que se cursan en cada institución de Educación Superior responden, en lo fundamental, a los lineamientos del desarrollo económico y social del país, las demandas que se identifiquen, y las capacidades y posibilidades de las instituciones de Educación Superior ${ }^{12}$. Igualmente, el plan de estudio se elabora para cada carrera

reemplazo acorde con su perfil profesional y científico, asegurando su continuidad y relevo cuando resulte necesario. Garantizar la adecuada atención al desarrollo de los jóvenes talentos en su vida laboral.

El término "extensión" expresa el conjunto de actividades, que no correspondan a la docencia ni a la investigación, relacionadas con la difusión cultural (científico y artística), transferencia de información hacia y desde la comunidad, consultorías u otros servicios, actividades de desarrollo comunitario, y, en general, todas las actividades de producción de bienes y servicios que realiza la institución mediante las relaciones interactivas con la comunidad, nación y región.

9 Publicada en Gaceta Oficial Ordinaria No. 25 de 21 de junio de 2018.

$10 C f r$. artículo 1 de la Resolución No. 2 de 2018 del Ministerio de Educación Superior.

11 Cfr. Idem.artículo 2.

$12 C f r$. Ibíd.artículo 7, segundo párrafo. 
en correspondencia con las necesidades existentes en el país, los avances científicos y tecnológicos de la época actual y las particularidades de la profesión en cuestión ${ }^{13}$.

El cumplimiento de los objetivos generales que se establecen en los planes de estudio de las carreras se logra mediante la ejecución del proceso docente-educativo. Se garantiza así, de conjunto con las actividades extracurriculares, la calidad de la formación de los futuros profesionales para satisfacer las exigencias sociales ${ }^{14}$.

En Cuba las instituciones de educación superior firman convenios con las entidades laborales y unidades docentes acreditadas, en que se establezcan con precisión los compromisos asumidos por cada una de las partes, en relación con la realización de las prácticas laborales y otras tareas afines que respondan a los vínculos de la universidad con la sociedad ${ }^{15}$. El control de la preparación adquirida por los egresados tiene como objetivo comprobar el grado en que los profesionales graduados por los centros de educación superior satisfacen los requerimientos de la sociedad ${ }^{16}$.

\section{HACIA UNA NUEVA RELACIÓN UNIVERSIDAD - SECTOR PRO- DUCTIVO}

Al entender que la Universidad es una organización abierta que interactúa con el medio circundante, se reconoce las influencias que recibe del exterior y las que de su interior parten hacia él. En un análisis tradicional, la relación comprende el otorgamiento de presupuesto por parte del Estado a la Universidad. La casa de altos estudios ofrece productos profesionales y servicios con el sector productivo, a la vez que el Estado regula la concentración de riqueza mediante los impuestos a los sujetos incluidos en ese sector.

Como se ha dicho anteriormente, los recursos de la Universidad en Cuba derivan de los fondos que el Estado le asigna a través del presupuesto nacional. A su vez, la Universidad retribuye por medio de los profesionales que egresan de ella y a través de los servicios de extensión universitaria, en general gratuitos, que brinda. La dinámica actual ha ido modificando ese esquema por otro que se puede que abarca el intercambio de los productos profesionales y servicios entre la Universidad y el sector productivo; mientras que el Estado también demandará de las potencialidades de esos sujetos económicos.

De este modo, la Universidad debe generar recursos propios para compensar los fondos que le proporciona el Estado. Su relación con el medio no se limita a la formación de los futuros profesionales ni a las actividades de extensión, sino que surgen como fuentes de recursos las actividades de transferencia de tecnología y conocimientos que puede realizar.

Esta realidad plantea la necesidad de una nueva relación entre Universidad y sector externo, institución que se debe constituir en un participante activo de la ge-

\footnotetext{
$13 \quad$ Cfr. Ibid. artículo 21.

14 Cfr. Ibid.artículo 124.

15 Cfr. Ibid.artículo 139

16 Cfr. Ibid.artículo 119.
} 
neración de conocimiento para el desarrollo. Su relación con el medio debe dar lugar a una interacción que no sólo permita difundir conocimiento de adentro hacia fuera, sino que debe permitir una retroalimentación de esa expansión cognitiva generada en el ámbito universitario.

"El papel de las universidades para colaborar en la construcción de una economía del conocimiento implica necesariamente que exista un sector productivo que aproveche el conocimiento y que esté dispuesto a establecer alianzas con instituciones académicamente autónomas. Al crearse tales alianzas, el sector privado debe mostrarse con mayor capacidad de iniciativa. Asimismo, debe tenerse en cuenta que el sector productivo contiene una parte pública y otra social que deben ser atendidas por la universidad (...) El mejoramiento de la calidad de las funciones sustantivas de las universidades debe estar asociado al incremento de la vinculación con los diversos tipos de empresas y necesidades de los sectores sociales" ${ }^{17}$.

Esta relación debe permitir que la Universidad amplíe sus fronteras de conocimientos, incorporando las problemáticas de su territorio. El contacto con su ambiente cercano debe servir para que los contenidos de sus planes de estudios se enriquezcan con las demandas de la sociedad en general. Debe existir un aprovechamiento social de la generación del conocimiento a fin de que la Universidad adquiera legitimidad social. Debe contar con iniciativa propia, a fin de producir las transformaciones necesarias para convertirse en referente de los cambios que la comunidad reclama.

Las transformaciones de la Educación Superior implican la apertura a un diálogo permanente con los distintos sectores de la sociedad, una conducta innovadora y flexible, vocación de servicio, respeto por los valores trascendentes y un fuerte compromiso con el destino mismo de sus naciones. Debe existir pertinencia social en la Educación Superior, a fin de que brinde servicios adecuados a su entorno. Resulta necesario articular las relaciones entre Universidad, Estado, sector productivo y comunidad. La forma en que se de esa articulación, tendrá que ver con una visión amplia de todos los sectores de la sociedad y con una firme decisión del Estado de jerarquizar el conocimiento y la Educación Superior como motores del desarrollo.

Los problemas que genera la escasez de fondos por parte del Estado a la educación superior ha dado lugar a diversos planteos sobre la necesidad de conseguir financiamiento alternativo. Es en este modelo donde la relación Estado-Universidad-sector productivo debe constituirse en un requisito estratégico para el desarrollo nacional. En esta relación, el Estado debe tener siempre presente las demandas de los actores sociales y la Universidad tiene que acercarse al sector productivo con sentido innovador y creativo.

Por otro lado, dada la importancia que para el desarrollo del país tiene el sector productivo relacionado con la pequeña y mediana empresa, es deber del Estado y de la Universidad atraer su atención, a fin de hacer viable su participación en la alianza estratégica que se debe dar entre estos actores sociales. La Universidad debe dejar de

17 H. H. HERNÁNDEZ BRINGAS, et. al., "Los desafíos de las universidades de América Latina y el Caribe ¿Qué somos y a dónde vamos?”, Perfiles Educativos, Vol. 37, No. 147, 2015, p. 211. 
lado cierto aislamiento intelectual para constituirse en generadora de conocimientos y de innovación tecnológica, ahora también orientada al sector de las pequeñas y medianas empresas y a las necesidades sociales. Debe existir un aprovechamiento social del conocimiento que se genere en las Universidades. En un proyecto de país que tenga al Estado y al pueblo en una construcción conjunta, la casa de altos estudios debe asumir un papel activo, pero debe ser una Universidad flexible y adaptable a los cambios del entorno.

"Es por ello que el papel de las universidades es de suma importancia en los procesos de transformación de los países. Es en ellas donde se modelan las personas altamente capacitadas que las nuevas circunstancias demandan. Ahi se forman individuos no sólo preparados en la producción eficiente de haberes y saberes, sino comprometidos con el desarrollo de su país y con el de la humanidad (...) Los retos son enormes cuando se tiene plena conciencia de que una de las funciones de la educación superior es contribuir a la movilidad social y al mejoramiento de las condiciones de vida de la población (...) El carácter público obliga, además, a la transparencia y la rendición de cuentas a la sociedad"18.

\section{EL MODELO DE LA TRIPLE HÉLICE Y LOS VÍNCULOS UNI- VERSIDAD-GOBIERNO-SECTOR PRODUCTIVO}

Los investigadores Henry ETZKOWITZ y Loet LEYDESDORF han estudiado los vínculos que se están produciendo entre estos tres actores, y generaron una propuesta teórica de estas relaciones en un concepto que denominan "la Triple Hélice" ${ }^{19}$. Esta se constituye una plataforma de formación institucional, en la cual la Universidad, la industria y el gobierno forman una relación recíproca que propende por mejorar el funcionamiento de las partes.

El modelo identifica una primera hélice compuesta por las relaciones entre las Universidades y los entornos científicos; la segunda, compuesta por las empresas y las industrias y la tercera, por las administraciones o los gobiernos. De la interacción y vasos comunicantes entre estas hélices se desarrolla innovación en un proceso complejo con roles a veces difusos y cambiantes, pero que genera la congregación del potencial para el conocimiento innovador, los recursos económicos y las posibilidades de mercado y las normas e incentivos de las políticas de innovación.

ETZKOWITS define la nueva misión de la Universidad como la que está encaminada a facilitar al acceso al conocimiento y a constituirse además como un actor económico fundamental. La Universidad emprendedora ${ }^{20}$ por lo tanto se basa en cuatro pilares: liderazgo académico capaz de formular e implementar una visión

18 H. H. HERNÁNDEZ BRINGAS, et. al., "Los desafíos...”, cit., p. 205.

19 H. ETZKOWITZ y L. LEYDESDORFF, The dynamics of innovation: from national systems and "mode 2" to a triple helix of university-industry-government relations, 2000, (accessible en: http://users.fmg.uva.nl/lleydesdorff/rp2000/, consultado el 5 de enero de 2018).

20 El término original en inglés la denomina como entrepreneurial university, lo que puede ser traducido como Universidad empresarial o emprendedora. El autor de esta investigación ha de- 
estratégica; control legal sobre los recursos académicos (propiedad física e intelectual); capacidad organizacional para transferir tecnología a través de patentes, licencias y un ambiente emprendedor entre funcionarios administrativos, profesores y estudiantes.

Este tipo de Universidad se caracteriza por su capacidad de gestionar sus propios recursos y su capacidad emprendedora le brindan las herramientas de negociación a profesores, estudiantes y administrativos para obtener provecho de las relaciones con las otras esferas. De igual forma, el liderazgo en investigación conlleva a que las mejores Universidades tengan mayor poder de negociación, por su aporte y los rendimientos que puede generar una asociación con ellas. Finalmente, el marco institucional y las herramientas jurídicas le permiten obtener la propiedad legal del conocimiento generado por esta y su posterior difusión a través de licencias o el otorgamiento de patentes.

De igual forma su capacidad empresarial le permite vincularse en la creación y asesoramiento de empresas, abriendo campo para una transferencia de conocimientos entre los actores económicos y la academia, lo que genera nuevas fuentes de financiación ${ }^{21}$.

La Universidad ostenta los roles tradicionales de educación e investigación, pero la capacidad de emprendimiento le permite generar cooperación e inclusive competir con empresas que se enfoquen en procesos de investigación y desarrollo. La posibilidad de establecer sus lineamientos estratégicos y participar con otras esferas institucionales en términos iguales, desemboca en que su contribución en proyectos para el desarrollo y el crecimiento económico refleje la importancia de su aporte. De esta forma, la dirección que asuma la Universidad y los proyectos de emprendimiento dependerán de los valores que la institución y la sociedad desee realizar.

La sinergia que producen estas tres hélices lleva al desarrollo de investigación polivalente con un potencial simultáneo en campos teóricos, tecnológicos y comerciales. Las Universidades a través de los vínculos con las otras dos esferas mejoran el acceso de los estudiantes y los profesores a nuevos problemas y campos científicos e industriales; promoviendo la investigación aplicada y la formulación de nuevas preguntas con potencial teórico. Adicionalmente, el influjo de nuevos recursos y la coordinación con las necesidades y conocimientos de la industria, potencializa las capacidades de la Universidad para generar avances en este campo.

Por supuesto existen objeciones a que la Universidad entre en contacto con otras esferas y que como resultado pierda su función principal de enseñanza, pero es la posibilidad de formular novedosos problemas de investigación la que repercute positivamente en la calidad de la educación. Adicionalmente, los conflictos de intereses que surgen de un modelo trilateral crean desafíos a cada una de las partes para reevaluar su posición en la sociedad e implementar estrategias que renueven y opti-

cidido el empleo de la segunda acepción pues, el término en inglés hace referencia adicionalmente a la capacidad creativa y no sólo a la característica de empresa.

21 La Universidad Nacional de Singapur y el Instituto Avanzado de Ciencia y Tecnología de Corea pueden citarse como ejemplos. 
micen sus funciones. De lo contrario, con una academia aislada de las necesidades de la sociedad y los actores económicos, puede resultar ser difícil de realizar.

La pregunta trascendental sigue siendo cómo acelerar el aporte de las Universidades en el desarrollo social y económico. Es consideración de esta investigación que un modelo educativo y de innovación, como el de la Triple Hélice, que realce la importancia de la educación universitaria en las estrategias para el desarrollo social y económico, y que acompañe al Estado y a las empresas en esta labor, puede convertirse en una respuesta adecuada a este tipo de desafío.

Los recursos económicos, que Cuba requiere para su desarrollo social y político, ya no pueden ser logrados solo dentro de los mercados internos. Es necesario participar en los mercados internacionales para lograr dichos recursos. Esto se logra con bienes y servicios de alta calidad y competitivos.

En una era informatizada, el porcentaje de las materias primas y la mano de obra en la estructura de costos es cada vez menor, a diferencia del valor agregado, o sea, el aporte intelectual que los recursos humanos suministran al bien o servicio en cuestión. Sobre esta base se dice que, la competitividad en los mercados no es otra cosa que la competitividad de los recursos humanos. Los recursos humanos nacionales deben tener los mismos patrones de formación que los recursos humanos de los competidores. De lo contrario la posibilidad de acceder y permanecer en dichos mercados es mínima.

El sistema de Educación Superior tiene una responsabilidad vital al respecto, ya que su ubicación al final del proceso de formación (docencia), exige que la investigación y la extensión garanticen el acabado y el mantenimiento de los recursos humanos con alta calidad. Como se evidencia, la calidad se alcanza cuando se satisfacen las necesidades de sus destinatarios.

Estas realidades, calidad, competitividad e informática exigen plantear la relación problema, objetivo y objeto en el contexto Universidad-sociedad con un enfoque sistémico, es decir, la casa de altos estudios se vincula mediante los problemas, que constituyen una necesidad social y son inherentes al objeto: relación Universidad-sociedad, al solucionarse, que se expresa esencialmente cuando el modo de actuación profesional se domine por estudiantes, docentes y profesionales del sector productivo-servicios mediante la práctica laboral investigativa común a los procesos: docencia, investigación y extensión, la Universidad cumple de modo más eficiente y eficaz el objetivo que se propuso.

La solución de los referidos problemas constituye la fuente fundamental del desarrollo de la motivación y creatividad de estudiantes, docentes, profesionales del sector de la producción y los servicios, y, en general, de la comunidad y sociedad; así como el elemento integrador de los componentes del objeto Universidad-sociedad, y especialmente en la cooperación Universidad-sector productivo y de servicios (estatal y privado).

Sobre la base de esta concepción cualquier cambio que se proponga hacer al sistema de Educación Superior, deberá orientarse a la formación de recursos humanos de calidad para vivir en una sociedad mejor. Al mismo tiempo, las instituciones 
universitarias son organizaciones complejas, cualquier cambio que se desee realizar deberá plantearse desde adentro y con previsión de sus posibles resultados.

Se considera que la práctica laboral investigativa es común a los procesos universitarios fundamentales: docencia, investigación y extensión; ya que es la forma fundamental de la expresión de la ley relación Universidad-sociedad y que se materializa a través de la comunicación social. Se pretende precisar el aspecto económico del objeto relación Universidad-sociedad, de modo que se pueda esclarecer la influencia de la educación superior en el desarrollo socioeconómico, particularmente de los países de América Latina en el contexto que se presenta en el mundo en lo que resta del presente siglo.

A la Universidad, como elemento vital del vínculo educación-sociedad, le es inherente una doble función de reproducción social y de innovación, en lo que parece ser la misión esencial de la educación, esto es, asegurar la continuidad y favorecer la renovación social.

A medida que el desarrollo socioeconómico depende cada vez más de la productividad del hombre y esta del nivel alcanzado en la formación, la educación superior es mayormente determinante del desarrollo socioeconómico. Más particularmente, cuando la capacidad de crear y adaptar las innovaciones tecnológicas determina el ritmo y orientación del desarrollo, la educación superior deja de ser un factor determinado, por el desarrollo económico para convertirse en determinante del mismo.

Resulta evidente la correspondencia entre los procesos del desarrollo socioeconómico - especialmente el área del trabajo, del empleo- y la formación brindada por la Universidad. Existe armonía entre el desarrollo universitario y las características que van adquiriendo las sociedades en sus procesos de innovación y aceleración, lo que constituye la condicionante exógena fundamental a consolidar para el exitoso resultado del cambio universitario y por ende, de los esfuerzos para lograr su dirección consecuente.

Las casas de altos estudios deben afrontar el reto de lograr la correspondencia más adecuada entre los resultados de sus acciones y los imperativos del desarrollo socioeconómico, orientando su potencial de inteligencia y creatividad al logro de mejores instituciones, al ser más eficientes y eficaces.

\section{A MODO DE CONCLUSIONES}

Se señala al conocimiento como uno de los factores estratégicos para el progreso de cualquier país y a la Universidad como el lugar que puede garantizar la generación participativa de conocimiento, a fin de contribuir al bienestar de toda la comunidad. Por lo tanto, es indudable la prioridad que debe tener el conjunto del sistema de Educación Superior en las decisiones que tome el Estado. Pero también es necesario que la Universidad deje de lado su estructura rígida, constituyéndose en una entidad flexible y versátil.

La necesidad de establecer una relación de cooperación entre la Universidad y el sector productivo no está en discusión, pero a partir del reconocimiento de la 
singularidad propia de la Universidad, que tiene un origen y un financiamiento esencialmente social. En este sentido, la relación debe ser más amplia que el simple contacto empresarial para abarcar la totalidad de los actores económicos que integran la sociedad en su conjunto.

El modelo de la Triple Hélice brinda un marco conceptual para entender los cambios que ocurren en la función social de las Universidades y los conocimientos científico-tecnológicos que generan. En este marco los actores económicos son miembros de la Triple Hélice como lugar de producción, el gobierno un promotor de relaciones contractuales que garantizan interacciones e intercambios estables y la Universidad es la fuente de conocimiento y tecnología; o como también se denomina, el agente provocador que permite la creación de sociedades basadas en el conocimiento.

De igual forma en una sociedad en vías de desarrollo como la cubana, el modelo brinda estrategias que pueden ser implementadas con suficiente libertad para que sus resultados se adapten a las necesidades locales.

En el caso específico de Cuba se conjugan elementos que podrían catalizar la generación de un modelo innovador fuerte. En primer lugar, la discusión que se está generando frente al tipo de modelo educativo brinda un espacio de discusión de propuestas enfocadas en el desarrollo de mejores profesionales y Universidades comprometidas con el desarrollo del país.

La Educación Superior debe ser eficiente en la utilización de sus recursos y racional en los gastos, pero el concepto de racionalidad propio de la empresa no es medida para la Universidad. Su rentabilidad, su eficiencia estará dada por las respuestas que pueda dar a las necesidades sociales. El fundamento de esta institución transita hoy por la capacidad de procesar la información y el conocimiento en función de las necesidades de la comunidad. En la alianza estratégica que se debe dar entre la Universidad y el sector productivo, resulta imprescindible establecer un flujo de comunicación habitual, puesto que la sociedad no se contenta con demandar de la Universidad su tradicional función docente. Al mismo tiempo, el sector productivo requiere el apoyo universitario en la generación y aplicación de conocimiento estratégico, específicamente de alto nivel, que contribuya decididamente en el proceso productivo. De esta manera se podrá generar una mayor actividad económica y una mayor riqueza que redundarán en beneficio de la sociedad cubana toda.

\section{BIBLIOGRAFÍA}

ACOSTA SILVA, A., "Políticas, actores y decisiones en las Universidades Públicas en México: un enfoque institucional", Revista de la Educación Superior, Vol. 42 (1), No. 165, enero-marzo, 2013, pp. 83-100.

ADROGUÉ DE DEANE, C., et. al., "La investigación en las universidades privadas de la Argentina. Cambios tras las políticas de aseguramiento de la calidad y financiamiento competitivo", REICE Revista Iberoamericana sobre Calidad, Eficacia y Cambio en Educación, Vol.12, No. 3, 2014, pp. 73-91. 
ANTÚNEZ SÁNCHEZ, A. F.,BRUZÓN VILTRES, C. J. y VELÁZQUEZBORGES, S. M., "Un análisis a partir de la constitución cubana sobre el ejercicio del autoempleo, incidencias en el nuevo relanzamiento en el modelo económico del siglo XXI", Iberoforum Revista de Ciencias Sociales de la Universidad Iberoamericana, Vol. 8, No. 15, enero-junio, 2013, pp. 98-130.

AROCENA, R. y SUTZ, J., La Universidad Latinoamericana del Futuro. Tendencias Escenarios - Alternativas, sin ed., México, 2000.

AROCENA, R y SUTZ, J., "La Universidad en las políticas de conocimiento para el desarrollo inclusivo", Cuestiones de Sociología, No. 12, 2015, (accesible en: http:// www.cuestionessociologia.fahce.unlp.edu.ar/article/view/CSn12a02, consultado el 10 de mayo de 2018).

BALL,S. J., "Globalización, mercantilización y privatización: tendencias internacionales en Educación y Política Educativa", Education Policy Analysis Archives, Vol. 22, No. 41, 2014, pp. 1-13.

ETZKOWITZ, H., The Triple Helix: University-Industry-Government Innovation in Action, Editorial Routledge, New York, 2008.

ETZKOWITZ, H. y LEYDESDORFF, L., The dynamics of innovation: from national systems and "mode 2 " to a triple helix of university-industry-government relations, 2000 (accesible en: http://users.fmg.uva.nl/lleydesdorff/rp2000/, consultado el 5 de enero de 2018).

FABELA-CÁRDENAS, M. A. y GARCÍA-TREVIÑO,A. H., "Gestión de la calidad educativa en educación superior del sector privado", Magis Revista Internacional de Investigación en Educación, Vol. 6, No. 13, enero-junio, 2014, pp. 65-82.

FLÓREZ-PARRA, J. M., "El gobierno corporativo en el sector público: Un estudio en las universidades públicas españolas", Cuadernos de Administración, Universidad del Valle, Vol. 29, No. 50, julio-diciembre, 2013, pp. 152-152.

FONTEOBA VIZCAÍNO,A., "Evaluación del riesgo y respuesta a los mismos en las entidades cubanas", Ekotemas Revista cubana de Ciencias Económicas, Vol. 1, No. 3, septiembre-diciembre, 2015, pp. 1-18.

GARCÍA-GUADILLA, C., "Universidad, desarrollo y cooperación en la perspectiva de América Latina”, Revista Iberoamericana de Educación Superior (RIES), Vol. 4, No. 9, 2013, pp. 21-33.

LA O SERRA, B., Decreto-Ley No. 305 De Las Cooperativas no Agropecuarias, Ediciones ONBC, La Habana, 2015.

MORALES RUBIANO, M. E., SANABRIA RANGEL, P. E. y FANDIÑO CUCA, F. A., "Estrategias de vinculación de los grupos de investigación con el sector productivo en el caso de la Universidad Nacional de Colombia”, Equidad \& Desarrollo, No. 20, julio-diciembre, 2013, pp. 143-165.

OJEDA SURÍS, D., "Las cooperativas no agropecuarias como modelo de gestión de los servicios en Cuba, un análisis de su creación y funcionamiento", Ekotemas Revista cubana de Ciencias Económicas, Vol. 1, No. 2, mayo-agosto, 2015, pp. 1-12. 
RIVERA RODRÍGUEZ, C. A., Manual para la organización, constitución y funcionamiento de las cooperativas, Centro de Estudios sobre Desarrollo Cooperativo y Comunitario. Asociación Nacional de Economistas y Contadores de Cuba, 2012.

RODRÍGUEZ MÉNDEZ,I. M., Consideraciones acerca del reconocimiento de los trabajadores por cuenta propia en Cuba, como empresarios mercantiles individuales, Tesis para obtener título de Licenciada en Derecho, Tutora de la Tesis MsC. Orelys Suárez Febles, Matanzas, Cuba, 2015.

ROMERO GÓMEZ, A., Transformaciones Económicas y Cambios Institucionales en Cuba, Centro de Investigación de la Economía Internacional, La Habana, 2014.

VELHO, L., VELHO, P.yDAVYT, A., "Las políticas e instrumentos de vinculación Universidad-Empresa en los países del MERCOSUR”, Educación Superior y Universidad, Vol. 9. No. 1, 1998, pp. 51-76.

VERGER, A., "Políticas de mercado, Estado y universidad: hacia una conceptualización y explicación del fenómeno de la mercantilización de la Educación Superior", Revista de Educación, No. 360, enero-abril, 2013, pp. 268-291.

VUOTTO, M. "Las cooperativas no agropecuarias y la transformación económica en Cuba: políticas, procesos y estrategias", REVESCO Revista de Estudios Cooperativos, No. 120, enero-abril, 2016, pp. 149-181. 